\title{
CT EVALUATION OF DISEASES OF PARANASAL SINUSES
}

\author{
Pushpa Ranjan ${ }^{1}$, Rajeev Ranjan ${ }^{2}$
}

${ }^{1}$ Senior Resident, Department of Radiology, IGIMS, Patna.

${ }^{2}$ Assistant Professor, Department of Surgery, NMCH, Sasaram.

\begin{abstract}
\section{BACKGROUND}

Diseases of paranasal sinuses include wide-spectrum ranging from inflammatory condition to neoplasm. CT has replaced conventional radiographs as imaging modality of choice for assessment of paranasal sinus diseases. In present study, thirty patients were taken for evaluation by CT referred by ENT Dept. The examination of paranasal region by CT was tailored according to individual clinical problem, covering overall area to make the study anatomically complete. This study proved good results of CT evaluation of diseases of paranasal sinuses due to high sensitivity and specificity to diagnosis and the planning of manageme nt in paranasal sinus diseases.
\end{abstract}

\section{KEYWORDS}

Paranasal Sinus, Maxillary Sinus, CT Evaluation.

HOW TO CITE THIS ARTICLE: Ranjan P, Ranjan R. CT evaluation of diseases of paranasal sinuses. J. Evolution Med. Dent. Sci. 2016;5(67):4843-4850, DOI: $10.14260 /$ jemds/2016/1102

\section{INTRODUCTION}

Computed tomography entered the medical imaging arena in 1970s with an abruptness, which is perhaps unparalleled in the history of medical imaging. Indeed, so successful has CT become that it effectively replaced many other radiological procedure.

CT is accepted as the gold standard for pathologicalanatomical evaluation of paranasal sinus disease.(1) In addition, it identified erosive process and acquired developmental deficiencies of bone.(2) Reducing scanning time and radiation dose of paranasal sinus, MDCT plays an important role. CT is accepted as the gold standard for pathological-anatomical evaluation of paranasal sinus disease.(3) CT is especially considered an obligatory part of planning surgical procedure.(4) In addition, it identified erosive process and acquired developmental deficiencies of bone. Reducing scanning time and radiation dose of paranasal sinus, MDCT plays an important role.

\section{MATERIAL AND METHOD}

The present prospective study was done in the Department of Radiodiagnosis, Darbhanga Medical College and Hospital, Laheriasarai. A total of thirty patients with suspected diseases of paranasal sinuses were evaluated by computed tomography. The cases were referred by the Department of Otolaryngology of this Institute.

\section{Method of Data Collection}

1. History: Detailed chronological history was obtained about development of disease in paediatric cases antenatal and perinatal history was also included.

2. Physical Examination: Comprising systemic and local examination.

Financial or Other, Competing Interest: None.

Submission 15-07-2016, Peer Review 07-08-2016,

Acceptance 13-08-2016, Published 22-08-2016.

Corresponding Author:

Dr. Pushpa Ranjan,

Room No. 6.

New MDH, IGIMS,

Sheikhpura, Patna-14.

E-mail: pushpa.rajeevranjan@gmail.com

DOI: $10.14260 /$ jemds/2016/1102
Local examination of organ was done of - chronic headache epistaxis, nasal discharge, local tumour in case of paranasal sinus diseases.

\section{Pathological Examination}

a. Haematological investigation - complete blood count, BT, CT, Blood Sugar, $\mathrm{T}_{3}, \mathrm{~T}_{4}$, TSH.

b. Fungal swab.

c. Histopathological investigation.

4. Radiological Examination

a. X-ray PNS.

b. CT scan of PNS.

CT evaluation of all these cases was done in CT scan unit of Department of Radiodiagnosis, Darbhanga Medical College and Hospital, Laheriasarai. Patients were evaluated by Siemens Somatom SP IVth Generation Spiral CT Scanner.

\section{Preparation of Patient}

Informed consent is taken from patient or his attendant. Many objects including dentures, jewellery, hairpins, eyeglasses should be removed that may affect CT image. If IV contrast is used, then we tell patient not to eat or drink anything 4-5 hours before the CT scan is done and also take history of allergy to IV contrast, any recent illness or systemic diseases, e.g. kidney diseases, diabetes, cardiovascular disease, etc., if present should be treated before CT scan done.

\section{Patient Position}

The plane for CT scanning of paranasal sinuses was kept parallel to inferior orbitomeatal line (Reid's baseline) starting at the level of maxillary alveolus just below the hard palate and to end above the level of frontal sinus. ${ }^{(5)}$ These axial scans were reviewed to determine the necessity of coronal sections to evaluate structures that are parallel to inferior osteomeatal line plane. Contiguous thin sections of $5 \mathrm{~mm}$ thickness in both planes and $3 \mathrm{~mm}$ thickness in area of interest taken.

1. Axial (Supine) and

2. Coronal (Prone)

Were routinely contemplated in majority of the cases. 
- $\quad$ Scanner settings - kVp 110/MAS 83.

- Sedation - was required for satisfactory study in infants and children syrup tricloryl orally was used in appropriate dose and in older patients (Inj. Largactil/calmpose, intravenously) who were unable to co-operate.

- Contrast - intravenous iodinated contrast media used in CT scanning consists of both Ionic and Non-Ionic variety.

Ionic Contrast Commonly used Include

1. Trazograf $-76 \%$ (adult) or $60 \%$ (children).

2. Urografin $-76 \%$ (adult) or $60 \%$ (children).

\section{Non-Ionic Contrast used Include}

1. Omnipaque

2. Ultravist

Now-a-days non-ionic contrast media are used. IV contrast media injected in dose of $1-2 \mathrm{~mL} / \mathrm{kg}$ body weight before obtaining the scan.

The examination of paranasal region by CT was tailored according to individual clinical problem covering overall area to make the study anatomically complete. It was essentially ensured that patient's head positioning be symmetrical and to remain so without movement during scanning.

After completion of CT scanning, patient was taken to observation room and pulse, BP and respiration was observed.

Pathology Sinusitis: Headache, facial pain and swelling and nasal discharge are symptoms of infective sinusitis.

The primary CT findings in the sinus cavities are relatively uniform, mucosal thickening of at least $6 \mathrm{~mm}$.(6) As an intermediate stage, an air fluid level may be observed before the destructed sinus becomes totally opacified. The mucosal thickening of infective sinusitis shows contrast enhancement on CT and where the secretions are retained, multiple concentric rings appear in the affected sinus.

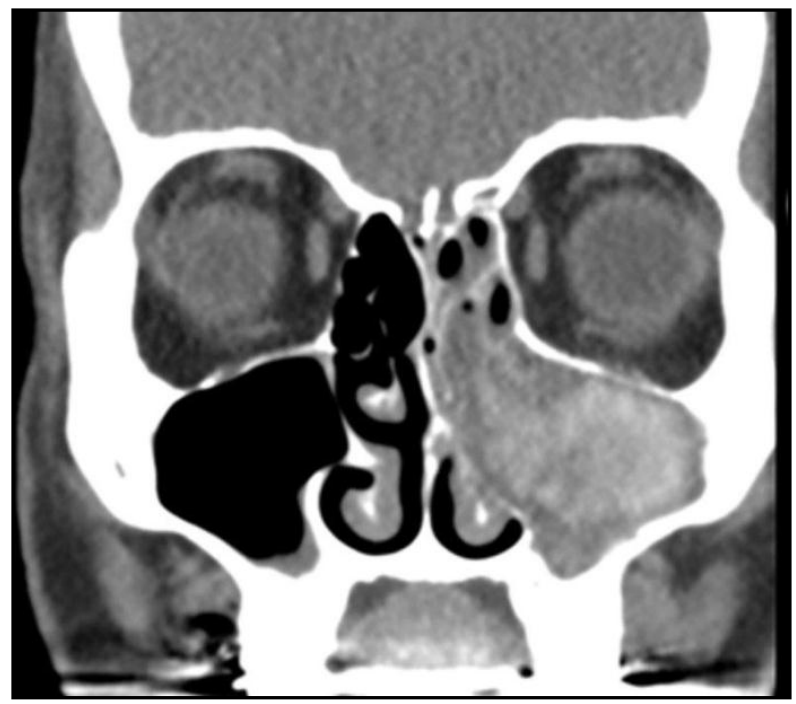

Fig. (1): Acute Sinusitis. Coronal CT Image show Mucosal Thickening and Opacification of Left Maxillary Sinus with Thick Secretion with Widening and Obstruction of Left Osteomeatal Complex. Mucosal Thickening also seen in Left Ethmoid and Frontal Sinus and Right Maxillary Sinus
Allergy may produce a spectrum of changes in paranasal sinuses varying from mild mucosal thickening to complete opacification of the sinus (Fig. 1). Thickening of nasal turbinates is characteristic accompanied by polypoid type of mucosal thickening producing a convex indentation into the cavity of the adjacent sinus. There is non-enhancement of hypertrophied mucosa. The condition is frequently complicated by nasal polyposis. Air-fluid levels are not usually seen in allergic sinusitis and when present the feature indicates superadded infection.

Clinically and radiographically, both allergic and bacterial sinusitis should show some improvement (documented by CT) after 2-3 weeks of conservative medical therapy. ${ }^{(7)}$

\section{Sino-Nasal Polyps}

Sino-nasal polyps are soft tissue pedunculated masses of oedematous hyperplastic upper respiratory mucosa. Commonest site is ethmoids followed by maxillary antra and then sphenoid sinus. Inflammatory polyps are indistinguishable from allergic polyp on imaging. CT displays homogeneous soft tissue characteristics with smooth, convex borders.(8) Multiple polyps in the ethmoid sinuses can be differentiated from tumour by demonstrating intact bony septa and a thin rim of mucoid material separating the polypoid masses.

The antrochoanal polyp originates from maxillary antrum and extends through the ostium to the nasal cavity and nasopharynx. These solitary, unilateral lesions accounts for 4$6 \%$ of all nasal polyps. CT imaging in coronal plane is best for delineating the size and location of such polyps (Fig. 2).

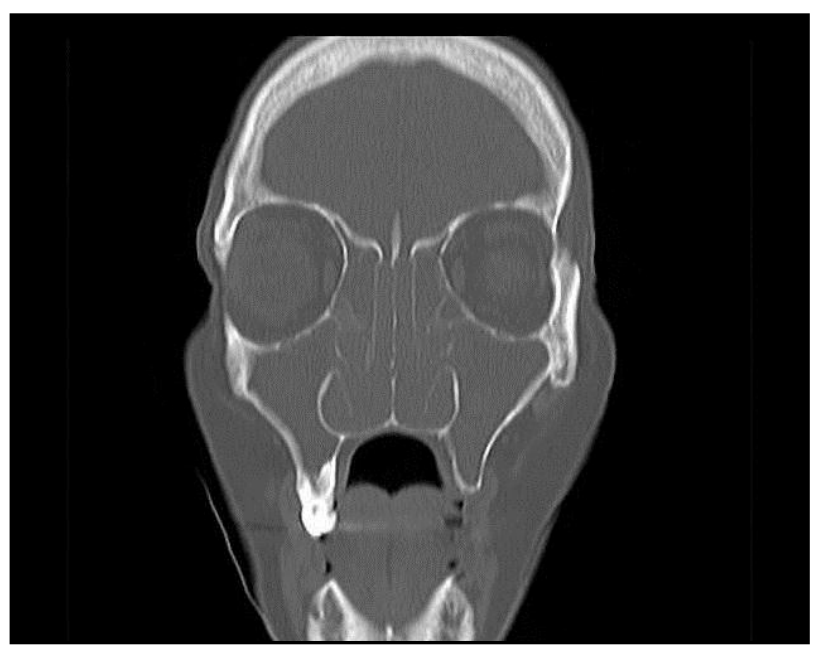

Fig. (2): Sino-Nasal Polyposis. Coronal CT Image shows Diffuse, Extensive, Mucosal Thickening and Soft Tissue Opacification of Bilateral Maxillary, Ethmoid, Frontal Sinuses. The Lesion Merges with Nasal Turbinates

\section{Mucocoeles}

Mucocoeles of the paranasal sinuses are benign, expansile paranasal sinus masses; with time they slowly enlarge to expand and erode bony structures. These lesions have been most commonly reported in the frontal $(66 \%)$ and ethmoid sinuses $(25 \%)$ and $10 \%$ in maxillary sinuses $(10 \%)$. Mucocoele of sphenoid sinus is rare.

The majority of mucocoeles have a homogeneous appearance on CT, and in general the lesions do not enhance with contrast infusion. 
These are most often isodense relative to brain tissue, but this may vary depending on whether they contain clear mucous or thick viscous material of a pyocoele.(9) Both transverse and coronal CT are essential for adequate evaluation.

Specific CT signs for mucocoele is described as an isodense mass with clear cut margins on orbital and cranial sides without signs of infiltration. A peripheral shell-like calcification is considered to be a remodelled bone or result of repeated attacks of infection in the mucocoele.(10)

Antral mucocoeles are well shown on axial scans, particularly demonstrating any extension into the nasal fossa. An advanced lesion may produce destruction of the maxillary antrum wall making differentiation from a carcinoma impossible.

\section{Benign Tumours}

Fibro-osseous lesions are very common incidental finding of the craniofacial bones are a challenging group of condition that is difficult to classify and treat. Conventional radiography and CT scanning show thickening of the facial skeleton with encroachment on the lumen of the maxillary sinus. CT characteristics of fibrous dysplasia include expansion of the involved bone with heterogeneous pattern of densities associated with scattered or confluent islands of bone formation.(11) Involvement of optic canals, fissures and osteomeatal complex have been best evaluated by CT (Fig. 3).

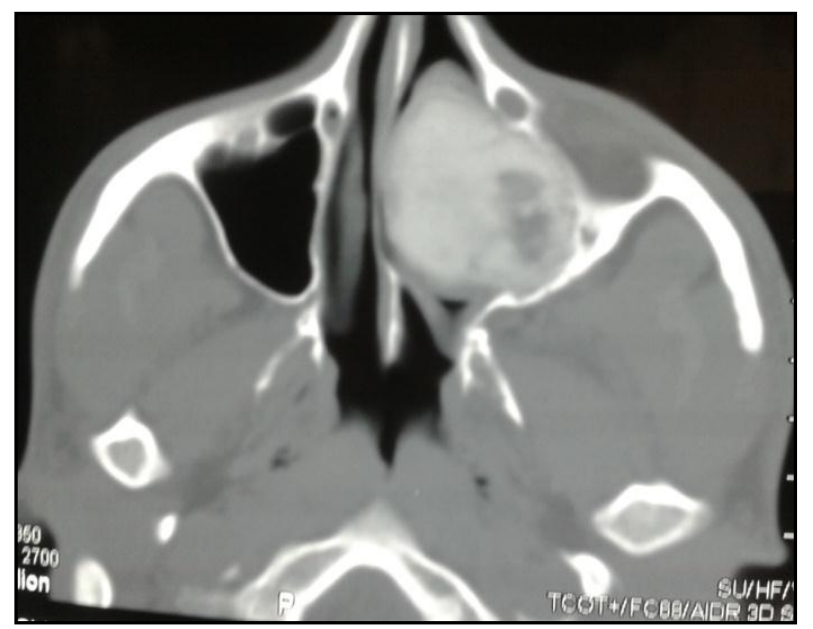

Fig. (3): Fibrous Dysplasia. Axial CT Image in Bone Window shows Hyperdense Bony Lesion seen in Left Maxillary Sinus

Angiofibroma is a benign, highly vascular, nonencapsulated neoplasm that occurs exclusively in male adolescents with intracranial involvement in 20 to $36 \%$ of patients.

CT is believed as better option to reveal the gross anatomy of these lesions than other imaging techniques. The geographic of the tumour is usually obvious even without contrast injection; however, enhanced scan should always be obtained in which the angiofibroma will demonstrate intense staining relative to the adjacent muscle.(12)

If diagnosis of juvenile angiofibroma is suspected then CT is usually the initial investigation in assessing the extent of tumour in the pterygoid fossa with resultant anterior bowing of posterior wall of the maxillary sinus. It becomes desirable prior to biopsy, which may result in extensive bleeding (Fig. 4).

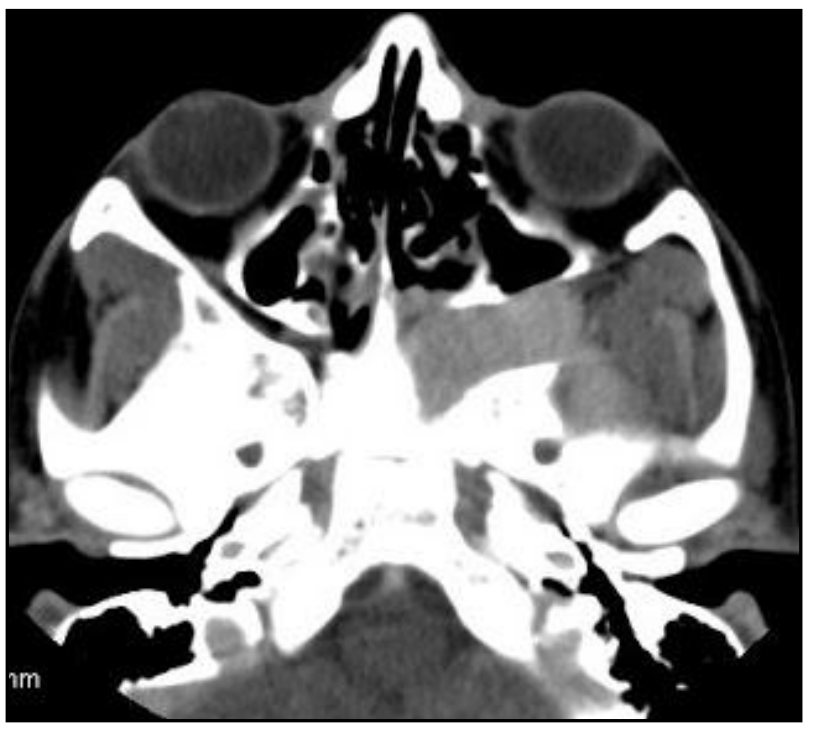

Fig. (4): Juvenile Nasopharyngeal Angiofibroma. Axial CT Scan of Paranasal Sinus show Enhanced Soft Tissue Lesion on Roof of Left Side of Nose involving Sphenopalatine Fossa Extending Superiorly to Involving Pterygopalatine Fossa causing Widening of it and Anterior Bowing of Posterior Wall Left Maxillary Sinus. The Lesion also Involve Left Temporal Fossa

\section{Osteoma}

Benign, slowly growing tumours contain mature compact or cancellous bone. They occur most frequently in frontal sinus followed by ethmoid and maxillary sinuses. CT findings are those of well-defined, very dense lesion if osteoma comprises compact mature bone, but an osteoma containing cancellous bone will be less ossified(13) Fig. 5 .

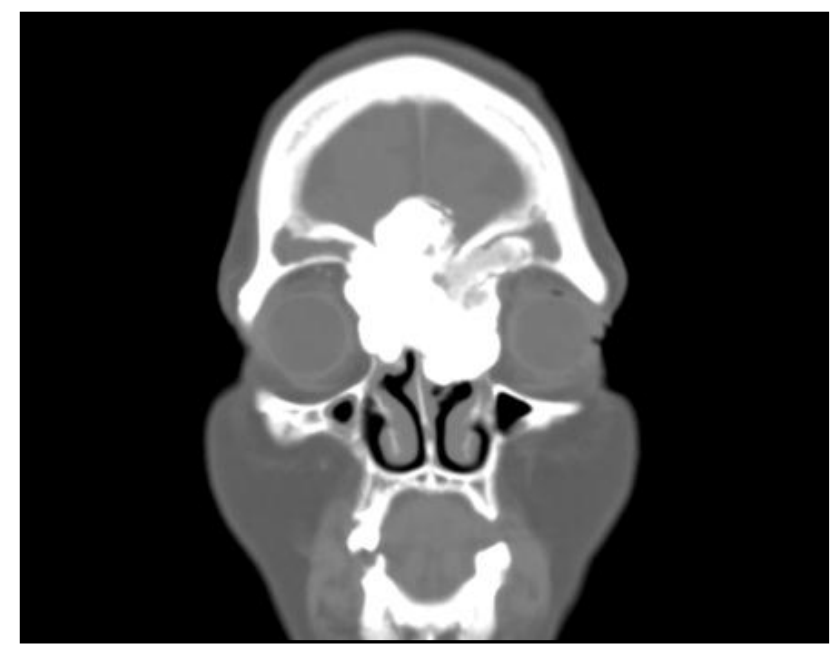

Fig. (5): Ivory Osteoma. Coronal CT Image on Bone Window shows Well-Defined, Hyperdense Lesion with Lobulated Border Involving Frontal Sinus, Bilateral Orbit and Anterior Cranial Fossa

\section{Papillomas}

Mucosal lining of nose and sinonasal cavity is derived from ectoderm and is called schneiderian membrane. This mucosa gives rise to three different types of papilloma - fungiform, inverted and cylindrical. Fungiform variety arises almost always from nasal septum, usually anteriorly.

The other two variety usually originate from lateral nasal wall. Inverted papillomas are commonest. Characteristically 
they arise from lateral nasal wall near the middle turbinate and extend into sinuses usually involving the maxillary and ethmoid sinuses.

The most common presenting symptoms are nasal obstruction, epistaxis and anosmia. It is important to differentiate papilloma from simple cyst and polyps of the sinonasal cavity, because the possibility of malignant degeneration within papilloma. They are locally invasive and are frequently multicentric and tends to recur after surgical excision.

Malignant Tumours: Malignancies affecting the paranasal sinuses are uncommon lesion, account for $0.2-0.8 \%$ of all CA and only $3 \%$ of those in the upper aerodigestive tract. They are frequently asymptomatic in the initial stages or produce symptoms indistinguishable from the more common disease process such as sinusitis. At diagnosis they are often at an advanced stage and have usually spread beyond in the site of origin, so carry a poor prognosis.

There are multiple risk factor for sinonasal cancer including exposure to hardwood dust (Adenocarcinoma) soft wood dust (Squamous cell carcinoma), nickel refining, chromium working, boot, shoe and textile working, volatile hydrochloride and snuff taking, human papilloma virus may be a cofactor. There is male-to-female predominance of $2: 1$ and secure primarily in $6^{\text {th- }} 7^{\text {th }}$ decade of life.

According to Sutton $7^{\text {th }}$ edition, squamous cell carcinoma account for $80 \%$ of all sinus malignancy. Approximately 50 $65 \%$ rise in maxillary sinuses, $10-25 \%$ in the ethmoid sinuses and $15-30 \%$ in the nasal cavity.

The CT diagnosis of a tumour requires the presence of a soft tissue mass, the enhancement characteristics of which can be useful especially when the tumour bulges into a sinus and obstructs sinus drainage rather than true invasion.(14) Most tumours are isodense or at least minimally enhancing masses, whereas mucous secretions usually exhibit low attenuation and are non-enhancing.

Because of similarity in squamous cell carcinoma and other carcinoma to adjacent secretions within obstructed sinus, a small region of bony abnormality and apparent destruction is important for early imaging diagnosis of carcinoma, especially squamous cell carcinoma. CECT used to differentiate between tumour and inflammatory mucosa, tumour themselves tend to enhance very little, whereas inflammatory mucosa may sometimes enhances brightly.(15) However, enhanced (Gd DTPA) MRI examination may be most useful for differentiating neoplastic from inflammatory mass within the paranasal sinuses when this diagnosis is in doubt.

\section{Lymphoma}

Most common sarcoma involving the sinonasal tract. Majority of these are non-Hodgkin's type of lymphoma. Non-Hodgkin's lymphoma is the $2^{\text {nd }}$ most common malignancy of head and neck following squamous cell carcinoma. It is important to recognize lymphoma as distinct from squamous cell carcinoma because of different clinical course and marked radiosensitivity of lymphoma relative to squamous cell carcinoma. Nasal cavity and maxillary sinus are most common site.

Lymphoma represents $8 \%$ of all paranasal sinus malignancies. On CT studies, lymphomas of the nose and paranasal sinus are seen as bulky masses and there may be changes to indicate expansion, erosion and infiltration.(16) Bone remodeling tends to occur. They usually originate in the nasal fossae and maxillary, occurring less in the ethmoid sinus.

Olfactory Neuroblastoma or Aesthesio-Neuroblastoma Uncommon tumour that originate from neural crest cells and arise from olfactory epithelium of nasal cavity (Fig. 6). The incidence has bimodal curve with first peak in $2^{\text {nd }}$ decade and second peak in $6^{\text {th }}$ decade. Epistaxis and nasal destruction are most common presenting symptoms. A unique feature regarding olfactory neuroblastoma is its unique site of origin and pathway of spread. On the other hand continued growth lead to extension into ethmoid and sphenoid sinuses and propensity for intracranial extension through dura in the region of cribriform plate.

On CT these tumours are homogeneous, enhancing masses that remodel the bone, commonly extending into ipsilateral ethmoid and maxillary sinuses and only rarely involve the sphenoid sinus. Calcification can occur

The presence of a tumour of the paranasal sinuses is detected on CT by demonstrating a soft tissue mass with or without involvement of the surrounding bone enclosing the paranasal sinus and nasal cavity. Categorizing the bone remodeling aids in differential diagnosis. The rapid permeating and destruction of bone is seen primarily with squamous cell carcinoma and some aggressive sarcomas. The type of contrast enhancement seen within this group of tumours of the paranasal sinuses varies from marked in angiosarcoma, through mild enhancement in rhabdomyosarcoma and most of the metastases to minimal or no enhancement with squamous cell carcinoma.

Gross bony erosion was easily recognized by CT, but minimal bony changes may not be demonstrated reliably as a result of partial volume effect, particularly in interpretation of anterior and medial antral wall defects.

CT with its excellent capability for displaying bone and soft tissue is the current diagnostic modality of choice for evaluating the osteomeatal complex. The development of functional endoscopic sinus surgery and its ability to alleviate disease in the osteomeatal unit allow treatment to be undertaken with minimal injury to adjacent normal structure. When the cause of recurrent or chronic inflammation can be readily identified on endoscopy, CT provides additional information regarding regional microanatomic detail, drainage impairment and the magnitude of disease.

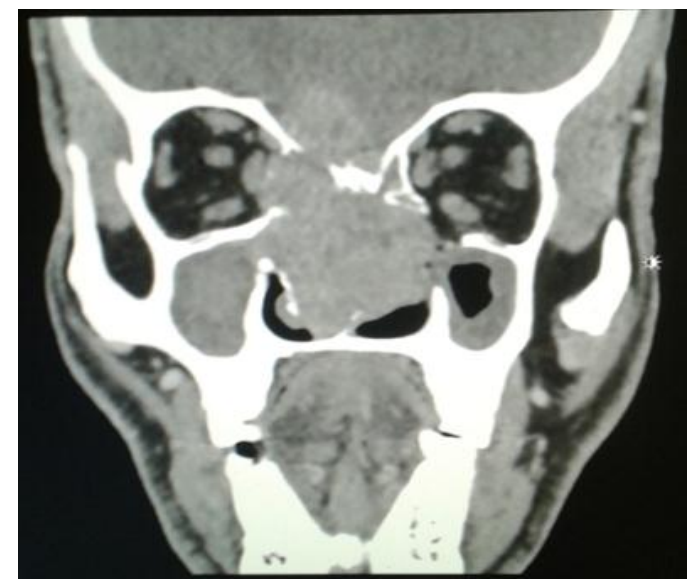

Fig. (6): Aesthesioneuroblastoma (Olfactory Neuroblastoma). Coronal CT Scan show Enhanced, Expansile, Locally Destructive Soft Tissue Lesion Occupying Ethmoid Air Cells and Surrounding Area Involving Right Orbit by Destroying Medial Wall and by Destroying Right Cribriform Plate Lesion Enter Into Anterior Cranial Fossa. Observations 


\begin{tabular}{|c|c|c|}
\hline Age Range (Years) & Male & Female \\
\hline $0-9$ & 2 & 1 \\
\hline $10-19$ & 7 & 2 \\
\hline $20-29$ & 2 & - \\
\hline $30-39$ & 1 & 4 \\
\hline $40-49$ & 4 & 2 \\
\hline $50-59$ & 3 & - \\
\hline $60-69$ & 1 & 1 \\
\hline Total (\%) & $\mathbf{2 0}(\mathbf{6 6 . 7 \% )}$ & $\mathbf{1 0}(\mathbf{3 3 . 3 \% )}$ \\
\hline Table 1: Age and Sex Distribution of Studied Case of \\
Diseases of Paranasal Sinus (n=30)
\end{tabular}

Table-1 shows out of 30 cases there was male predominance [20 cases $(66.7 \%)]$ compared to female [10 cases $(33.3 \%)$ ] youngest 8 years old and oldest 68 years old.

\begin{tabular}{|c|c|c|}
\hline \multirow{2}{*}{$\begin{array}{l}\text { Imaging } \\
\text { Parameter }\end{array}$} & \multicolumn{2}{|c|}{ Imaging Plane } \\
\hline & Axial $(n=30)$ & Coronal $(n=20)$ \\
\hline Patient position & Supine & Prone \\
\hline Gantry angulation & Parallel to IOML & $\begin{array}{l}\text { Perpendicular to } \\
\text { IOML }\end{array}$ \\
\hline Extent to study & $\begin{array}{l}\text { From hard } \\
\text { palate through } \\
\text { frontal sinus }\end{array}$ & $\begin{array}{c}\text { From anterior } \\
\text { frontal sinus to } \\
\text { post. Sphenoid } \\
\text { sinus } \\
\end{array}$ \\
\hline Section thickness & $5 \mathrm{~mm} / 3 \mathrm{~mm}$ & $5 \mathrm{~mm} / 3 \mathrm{~mm}$ \\
\hline $\begin{array}{c}\text { Table } \\
\text { incrementation }\end{array}$ & $5 \mathrm{~mm} / 3 \mathrm{~mm}$ & $5 \mathrm{~mm} / 3 \mathrm{~mm}$ \\
\hline
\end{tabular}

Axial scans were performed in all 30 cases and direct coronal scans in 20 cases to supplement the former, because in rest of the cases patients were not cooperative. Section thickness, radiographic factors and scan time were constant in both studies.

\begin{tabular}{|l|c|c|}
\hline \multicolumn{1}{|c|}{ Lesion } & No. of Patient & Percentage (\%) \\
\hline Inflammatory \\
\hline Sinusitis & 7 & 23.31 \\
\hline Mucocoele & 2 & 6.66 \\
\hline Neoplastic & 6 & 20.00 \\
\hline Carcinoma & 2 & 6.66 \\
\hline Sarcoma & 1 & 3.33 \\
\hline Lymphoma & 2 & 6.66 \\
\hline $\begin{array}{l}\text { Malignancy } \\
\text { unspecified }\end{array}$ & 5 & 16.65 \\
\hline Benign tumour & 4 & 13.32 \\
\hline Traumatic & 1 & 3.33 \\
\hline Miscellaneous & Table 3: Distribution of Clinical Material \\
\hline \multicolumn{2}{|c|}{ for Paranasal Sinus (n=30) } \\
\hline
\end{tabular}

- 30 cases were classified as showed in above table. There were 16 cases having neoplasia, of which 5 had benign lesions.

- In inflammatory group, in which 2 cases of mucocoele and 7 cases of sinusitis (which may be due to primary bacterial sinusitis, secondary sinusitis and allergic sinusitis).

\begin{tabular}{|c|c|c|}
\hline Involved Sinus & No. of Patients & Percentage (\%) \\
\hline Frontal & 4 & 13.3 \\
\hline Anterior Ethmoid & 16 & 53.3 \\
\hline Maxillary & 20 & 66.6 \\
\hline Posterior Ethmoid & 17 & 56.6 \\
\hline Sphenoid & 9 & 30.0 \\
\hline \multicolumn{3}{|c|}{$\begin{array}{l}\text { Table 4: CT Localization of Diseases of Paranasal Sinus } \\
\qquad(n=30)\end{array}$} \\
\hline
\end{tabular}

- Maxillary antrum was single most commonly involved site of disease (66.6\% of cases) followed by posterior and anterior ethmoid in $56.6 \%$ and $53.3 \%$ of cases respectively.

- Sphenoid and frontal sinuses were less commonly involved as demonstrated in CT scan of present study.

- Diseases of PNS are not confined to sinus of their origin, but they involve other sinus also ex. Ca of maxillary sinus also involve ethmoid sinus.

\begin{tabular}{|c|c|c|c|c|}
\hline $\begin{array}{c}\text { Age } \\
\text { Range }\end{array}$ & Primary & Secondary & Allergic & Mucocoele \\
\hline $0-9$ & - & - & - & - \\
\hline $10-19$ & - & - & 2 & 1 \\
\hline $20-29$ & - & - & 1 & 1 \\
\hline $30-39$ & 1 & - & - & - \\
\hline $40-49$ & 1 & 1 & - & - \\
\hline $50-59$ & - & 1 & - & - \\
\hline $60-69$ & - & - & - & - \\
\hline \multicolumn{5}{|c|}{ Table 5: CT Distribution of Inflammatory } \\
Disorder of PNS (n=9) \\
\hline
\end{tabular}

- Inflammatory disorder of paranasal sinus have classified in above table.

- There was predominance of primary sinusitis in age group of 30-49 years and prevalence of allergic sinusitis in 10-29 years of age group.

\begin{tabular}{|c|c|c|c|}
\hline $\begin{array}{c}\text { Findings } \\
\text { Mucosal } \\
\text { Changes }\end{array}$ & $\begin{array}{c}\text { Bacterial } \\
\text { Sinusitis } \\
(\mathbf{n = 2})\end{array}$ & $\begin{array}{c}\text { Allergic } \\
\text { Sinusitis } \\
(\mathbf{n = 3})\end{array}$ & $\begin{array}{c}\text { Secondary } \\
\text { Sinusitis } \\
(\mathbf{n}=2)\end{array}$ \\
\hline Unilateral & 1 & - & 1 \\
\hline Bilateral & - & 1 & - \\
\hline $\begin{array}{c}\text { Air-fluid } \\
\text { level }\end{array}$ & 1 & 1 & 1 \\
\hline $\begin{array}{c}\text { Polyp } \\
\text { formation }\end{array}$ & - & 1 & - \\
\hline \multicolumn{3}{|c|}{ Table 6: CT Features of Sinusitis of Paranasal Sinus (n=7) } \\
\hline
\end{tabular}

- Above table compare CT finding in three types of sinusitis.

- Polyp formation was feature of only in allergic sinusitis (1 case).

\begin{tabular}{|c|c|c|c|c|}
\hline \multirow{2}{*}{$\begin{array}{c}\text { Age } \\
\text { Range } \\
\text { (Years) }\end{array}$} & Male & Female & Male & Female \\
\cline { 2 - 5 } & - & 1 & - & 1 \\
\hline $0-9$ & 2 & - & 2 & - \\
\hline $10-19$ & 1 & 1 & - & - \\
\hline $20-29$ & - & - & 1 & 2 \\
\hline $30-39$ & - & - & 1 & 1 \\
\hline $40-49$ & - & - & 2 & - \\
\hline $50-59$ & - & - & 1 & - \\
\hline $60-69$ & 3 & $\mathbf{2}$ & $\mathbf{7}$ & $\mathbf{4}$ \\
\hline Total & $\mathbf{3}$ & \multicolumn{7}{|c|}{ Benign PNS (n=16) } \\
\hline \multicolumn{7}{|c|}{ Table 7: Age and Sex Incidence in Neoplasm } \\
\hline
\end{tabular}


- Above table shows the ratio of malignant and benign tumour as 2.2:1 in different age group.
- There was male predominance in case of malignancy as compared to female patients.

\begin{tabular}{|c|c|c|c|c|}
\hline Features & Carcinoma $(n=6)$ & Sarcoma $(n=2)$ & Lymphoma (n=1) & Malignancy Unspecified (n=2) \\
\hline \multicolumn{5}{|l|}{ Soft Tissue Mass } \\
\hline Homogeneous & 4 & - & 1 & 1 \\
\hline Heterogeneous & 2 & 2 & - & 1 \\
\hline Bone Destruction & 5 & 2 & 1 & 2 \\
\hline Sclerotic Reaction & - & 1 & 1 & - \\
\hline \multicolumn{5}{|c|}{ Contrast Enhancement } \\
\hline Positive & 2 & 2 & - & 1 \\
\hline Equivocal & 1 & - & 1 & - \\
\hline \multicolumn{5}{|c|}{ Table 8: CT Characteristics of Malignant Tumours of PNS $(n=11)$} \\
\hline
\end{tabular}

- Above table compare the CT features of carcinomas, sarcomas, lymphomas and unspecified malignancies.

- Bone involvement was commonly present in all the tumours.

\begin{tabular}{|c|c|c|}
\hline Type of Extension & No. of Cases & Percentage (\%) \\
\hline Intranasal & 06 & 66.66 \\
\hline Intracranial & 02 & 22.22 \\
\hline Intraorbital & 01 & 11.11 \\
\hline \multicolumn{2}{|c|}{ Table 9: Demonstration of CT Extension } \\
of Malignant Lesion of PNS (n=9)
\end{tabular}

- $\quad$ Among 11 cases of malignancy of PNS, here extension of 9 cases are shown because 2 cases are localized to their origin.

- Above study showed most of the tumour of various PNS, has tendency to extend intranasally.

- Intracranial extension is more common than intraorbital extension.

\section{DISCUSSION}

Thirty patients with various disease entities of paranasal sinus region were studied to evaluate site of the lesion, extent of spread and aetiopathologic diagnosis as indicated by CT characteristics.

The present study include 20 male and 10 female patients. Patient with minimum age was 8 years old and maximum age of 68 years old.

In cases of tumours of PNS which extend into orbits, coronal views are supplementary to axial scans, whereas a sphenoid or ethmoid lesion extent laterally. Extension into the orbital roof or cribriform plate however can only be evaluated on coronal scan. This is also true for maxillary sinus tumour extending into the orbital floor.

There were 16 cases of neoplasia, of which 5 (16.6\%) had benign lesion and carcinoma ( 6 in no. $20 \%$ ), sarcoma ( 2 in no. $6.6 \%$ ), lymphoma (1 case, $3.3 \%$ ), unspecified malignancy (2 in no. 66\%) [Table 3].

Among sarcomatous lesion (2 in no.), maxillary antrum was involved in one case while ethmoids in another cases. CT feature include aggressive permeation of sinus walls, inhomogeneous enhancement pattern with residual bone fragments.

Solitary case of lymphoma is seen in present study, which is seen in maxillary antrum. Lesion are seen as bulky mass. CT shows changes to indicate expansion, erosion of sinus wall and equivocal enhancement. Primary malignant lymphoma of PNS is uncommon.

Maxillary sinus antrum was single most common involved site of disease (66\% of cases) followed by posterior and anterior ethmoid in $56.6 \%$ and $53.3 \%$ of cases respectively. Sphenoid sinus and frontal sinuses were less commonly involved as demonstrated in CT scan of present study.

Nine patients with inflammatory disorder of PNS, of which 2 (6.66\%) cases are of mucocoele and 7 (23.3\%) cases of sinusitis. Incidence of age prevalence of primary sinusitis in present study was 30-49 years and of allergic sinusitis in 10-29 age group when the sinus mucosa is seen in CT, it is pathologically involved. If the mucosal lining was nearly uniform, smooth, thickened appearance involving most or all of the sinus walls, it usually represents either inflammatory thickening or fibrotic scarring secondary to bacterial or allergic disease.

CT finding to sinusitis of PNS were categorized according to cause [Bacterial sinusitis (2), allergic sinusitis (3) and secondary sinusitis (2)]. Mucosal damage were noted as unilateral in 2 cases and bilateral in 1 case. Bacterial sinusitis usually involved each sinus independently. There are also some swelling of nasal cavity mucosa and the turbinates, but primary finding are in maxillary antra.

Allergic sinusitis considered as systemic process and as a result there is tendency for symmetrical sinus involvement and pansinusitis. Polyposis is often seen in association with sinus disease. Sometimes presence of polyp may have no clinical significance, since it may be found as an incidental finding [Table 6].

In age and sex distribution of neoplasm of PNS, there was male predominance as compared to female patient [Table 7].

CT characteristic of malignant tumours of PNS, comprising of carcinoma $(6,20 \%)$, sarcoma $(2,6.66 \%)$, lymphoma $(1,3.33 \%)$, unspecified malignancy $(2,6.66 \%)$ [Table 8].

Bone destruction was seen in carcinoma, sarcoma and unspecified malignancy, whereas sclerotic reaction/bone remodeling was seen in lymphoma. In present study, 4 cases of carcinoma showed homogeneous and 2 cases revealed heterogeneous soft tissue mass.

All 2 cases of sarcoma are heterogeneous. Lymphoma showed homogeneous enhancement. Among unspecified malignancy, 1 case showed homogeneous and another 1 case showed heterogeneous enhancement. The highly cellular masses have a homogeneous appearance.

Contrast enhancement was positive in 2 cases of carcinoma and 2 cases of sarcoma and 1 case of unspecified 
malignancy. Since most PNS tumour were avascular and blood brain barrier playing no part, IV contrast intravenous enhancement was of little value in further definition. However, it is possible that some vascular lesion may be enhanced.

CT extension of malignant lesion of PNS in 9 cases, 2 cases have localized malignancy to their origin. Most of tumour of PNS has tendency to extend intranasally (66.66\%); intraorbital extension (11.11\%) was less common than intracranial extension (22.22\%).

Despite the significant amount of information that can be gathered from a CT scan in the study of paranasal sinus tumours, several limitations exist:

- It cannot always determine whether tumour has invaded or just approached the periorbital region.

- It is difficult to differentiate tumour from soft tissue swelling, consequently to determine the degree of actual tissue invasion.

- It is seldom feasible to determine the extent of tumour infiltration into the obstructed sinuses because of similar soft tissue attenuated values of tumour and retained secretion.

\section{SUMMARY AND CONCLUSION}

The role of CT in study of paranasal sinuses was adjudged to demonstrate accurately the total extent of disease process and its potential nature. This was done to provide assistance in operative management and radiotherapy planning. The present study analysed 9 cases of inflammation, 4 of injury, 5 and 11 cases of benign and malignant neoplasms respectively, 1 miscellaneous case involving paranasal sinuses.

Visualization of sinus mucosa on CT proved to be the hallmark indicating its pathological involvement. Primary sinusitis affected a sinus independently, whereas there occurred a tendency for symmetrical sinus involvement in allergic sinusitis. Isolated posterior ethmoid clouding on CT scan may at time points towards dormant infected.

It was apparent that axial plane alone was not sufficient for adequate evaluation of 2 cases of mucocoeles. Coronal scan further characterized the orbital invasion (50\%) and the overall limit of this surgically curable non-neoplastic entity.

Coronal CT is now identified as the most effective means to demonstrate the microanatomic communications between anterior ethmoid sinus and the frontal and maxillary sinuses. The modality will be the further road-map to classify and localize the acquired or congenital aetiology of paranasal sinus obstruction, for functional endoscopic sinus surgery.

In benign neoplasms, CT added a little significant information to that obtained by conventional radiologic methods. In malignant lesions, however, CT provides a clear picture of the posterior, superior and orbital extent of paranasal tumours to be interpreted with potential accompaniment.

The histological incidence of malignancy in paranasal region confirmed to $45 \%$ carcinomas followed by $18 \%$ aggressive sarcomas. Two cases were lost in terms of cytological corroboration. Lymphoma is rare tumours, which cannot be differentiated radiologically from the carcinoma group.

The maxillary antrum is the most frequent site of tumour involvement, the next being ethmoid; however, more than one sinus cavity is often affected by tumour at the time of diagnosis.

Gross bone erosion was easily recognized on CT, but minimal osseous change may not be detected reliably as a result of the partial volume effect. In particular, if no soft tissue mass is discernible in either the underlying ethmoid cells or the adjacent orbit, such equivocal erosion is often not an area of pathological destruction.

Intravenous contrast material was used whenever possible in an attempt to differentiate inflammatory lesions from malignant tumours and tumours from obstructed sinuses. In majority of instances, it was sufficient to detect the differential enhancement amongst adjacent structures of paranasal region. Practically, mild degree of contrast take up and subtle calcification get virtually obscured, in widewindow CT images.

The most characteristic sign of malignancy is the loss of surrounding fascial planes due to tumour infiltration, discernable on CT scans. Extended of the soft tissue mass outside the normal confines of a sinus cavity was considered as indication for biopsy, which will remain the reliable mode for diagnosing malignancy.

Our review envisages the need of sheet anchor clinical background to supplement a CT study. Only with such approach can a secure differential diagnosis be offered and more importantly the CT evaluation is tailored to shell out imperative pathomorphologic details of interpretation.

The thesis has identified the diagnostic acumen of computed tomography for orbital and paranasal lesions, opening an investigative wide in this field without causing discomfort or risk to the patient of any age. A pre-requisite for early and accurate diagnosis is still a very close cooperation between ophthalmologist and the radiologist.

\section{REFERENCES}

1. Branstetter BF, Wissman JL. Role of MR and CT in the paranasal sinuses. Otolaryngol Clin N Am 2005;38(6):1279-99.

2. Perez-Pinas, Sabate J, Carmona A, et al. Anatomical variation in the human paranasal sinus region studied by CT. J Anat 2000;197(Pt 2):221-7.

3. Zinreich SJ. Functional anatomy and computed tomographic imaging of paranasal sinuses. American Journ of Med Sci 1998;316(1):2-12.

4. Lloyd GAS, Lund VJ, Scadding GK, et al. CT of paranasal sinus and functional endoscopic surgery: a critical analysis of 100 symptomatic patients. JLO 1991;105(3):181-5.

5. White PS, Cowan IA, Robertson MS. Limited CT scanning technique of paranasal sinus. JLO 1991;105(1):20-3.

6. Towbin R, Han BK, Kaufman RA, et al. Post septal cellulitis: CT in diagnosis and management. Radiol 1986;158(3):735-7.

7. Devaiah AK. Adult chronic rhinosinusitis: diagnosis and dilemmas. Otolaryngol Clin N Am 2004;37(2):243-52.

8. Scribano E, Blandino A, Ascenti G, et al. Computerized tomographic findings of benign disease of sphenoid sinus. Radiol Med 1998;95(5):409-16.

9. Riepen K, Lellig U, Schnepper U. Radiological diagnosis of inflammatory and tumour disorder of paranasal sinuses. Laryngorhinootologie 1989;68(10):543-6. 
10. Skora W, Kozlowaski Z, Dabrowska K, et al. Clinical evaluation of CT scan of nasal cavity and paranasal sinuses. Otolaryngol Pol 2008;62(5):553-7.

11. Som PM, Curtin HD. Chronic sinonasal diseases including benign tumors - the role of imaging. Radiol Clin North America 1993;31(1):33-44.

12. Aygun N, Uzuner O, Zinreich SJ. Advances in imaging of paranasal sinuses. Otolaryngol Clin N Am 2005;38(3):429-37.

13. Chopra H. Nasal tumors: a clinical, histopathological and radiological profile. Indian J Otolaryngol Head and Neck Surg 2008;60(2):112-6.
14. Chavda SV, Olliff JF. Text book of radiology and imagingDavid Sutton. $7^{\text {th }}$ ed. Chur Chill Living Stone 1519-29.

15. Lund VJ, Howard DJ, Lloyd GAS. CT evaluation in paranasal sinus tumours for craniofacial resection. The British J Radiol 1983;56(667):439-46.

16. Weber AL, Staton AC. Malignant tumours of paranasal sinuses: radiologic, clinical and histopathologic evaluation of 200 cases. Head and neck surg 1984;6(3):761-76. 